\title{
Partially acoustic dark matter, interacting dark radiation, and large scale structure
}

\author{
Zackaria Chacko, ${ }^{a}$ Yanou Cui, ${ }^{a, b, c}$ Sungwoo Hong, ${ }^{a}$ Takemichi Okui ${ }^{d}$ \\ and Yuhsinz Tsai ${ }^{a}$ \\ ${ }^{a}$ Maryland Center for Fundamental Physics, Department of Physics, University of Maryland, \\ Stadium Dr., College Park, MD 20742, U.S.A. \\ ${ }^{b}$ Department of Physics and Astronomy, University of California-Riverside, \\ University Ave, Riverside, CA 92521, U.S.A. \\ ${ }^{c}$ Perimeter Institute, 31 Caroline Street, North Waterloo, Ontario N2L 2Y5, Canada \\ ${ }^{d}$ Department of Physics, Florida State University, \\ College Avenue, Tallahassee, FL 32306, U.S.A. \\ E-mail: zchacko@physics.umd.edu, yanou.cui@ucr.edu, shong710@umd.edu, \\ okui@hep.fsu.edu, yhtsai@umd.edu
}

ABSTRACT: The standard paradigm of collisionless cold dark matter is in tension with measurements on large scales. In particular, the best fit values of the Hubble rate $H_{0}$ and the matter density perturbation $\sigma_{8}$ inferred from the cosmic microwave background seem inconsistent with the results from direct measurements. We show that both problems can be solved in a framework in which dark matter consists of two distinct components, a dominant component and a subdominant component. The primary component is cold and collisionless. The secondary component is also cold, but interacts strongly with dark radiation, which itself forms a tightly coupled fluid. The growth of density perturbations in the subdominant component is inhibited by dark acoustic oscillations due to its coupling to the dark radiation, solving the $\sigma_{8}$ problem, while the presence of tightly coupled dark radiation ameliorates the $H_{0}$ problem. The subdominant component of dark matter and dark radiation continue to remain in thermal equilibrium until late times, inhibiting the formation of a dark disk. We present an example of a simple model that naturally realizes this scenario in which both constituents of dark matter are thermal WIMPs. Our scenario can be tested by future stage-IV experiments designed to probe the CMB and large scale structure.

KeYwords: Cosmology of Theories beyond the SM, Beyond Standard Model

ARXiv EPrint: 1609.03569 


\section{Contents}

1 Introduction 1

2 A benchmark model 3

3 General evolution of DM density perturbations 5

4 Numerical results and corrections to the CMB spectrum 10

5 Conclusions $\quad 14$

\section{Introduction}

For nearly two decades, the $\Lambda \mathrm{CDM}$ paradigm in which dark matter (DM) is composed of cold, collisionless particles has provided an excellent fit to cosmological data. Although on galactic scales or smaller there have been long-standing issues such as the core-vs-cusp [1,2] and too-big-to-fail [3] problems that are difficult to explain within this framework, it has been very successful on larger scales. However, in recent years, as the data has become more precise, the $\Lambda \mathrm{CDM}$ framework has also come into tension with measurements on large scales. In particular, the value of today's Hubble rate $H_{0}$ obtained from a fit to the cosmic microwave background (CMB) and baryon acoustic oscillation (BAO) data [4] is smaller than the results from local measurements [5-9], with a $\sim 3 \sigma$ discrepancy. Similarly, the inferred value of $\sigma_{8}$ (the amplitude of matter density fluctuations at the scale of $8 h^{-1} \mathrm{Mpc}$ ) is larger by $3-4 \sigma$ [10-12] than the values from direct measurements such as weak lensing survey [13], CMB lensing [14], and Sunyaev-Zeldovich cluster counts [15-17].

These large-scale anomalies are particularly intriguing because the theoretical understanding of dynamics at large scales is rather simple and robust, essentially requiring only the application of linear perturbation theory to density fluctuations. This is in contrast to the studies of small scale structure, which not only require an understanding of the nonlinear evolution of a many-body system but also crucially depend on the detailed dynamics of baryons, which is quite challenging to simulate. Therefore, while it is possible that these large scale discrepancies are due to systematic errors in the associated experiments [18, 19], it is important to consider the possibilty that they are in fact robust problems that require a fundamental shift away from the $\Lambda$ CDM framework.

Several proposals have been put forward to explain one or the other of these anomalies by going beyond the $\Lambda \mathrm{CDM}$ paradigm. For example, DM that decays at late times, well after the CMB epoch, can reduce the size of $\sigma_{8}[20,21]$. Alternatively, neutrinos with masses near the top of the allowed range or sterile neutrinos with an $\mathrm{eV}$ mass can fit the $\mathrm{CMB}$ and $\mathrm{BAO}$ data with a smaller $\sigma_{8}$ [22], but have the effect of making the $H_{0}$ 
problem worse [4]. If there is dark radiation (DR) that behaves like a tightly coupled fluid (as opposed to free streaming like neutrinos), this can ameliorate the tension in $H_{0}$ measurements [23]. If DM further scatters with such DR, it may be possible to solve both the $H_{0}$ and $\sigma_{8}$ problems [24-27]. These proposals, however, require a rather tiny DM-DR interaction constrained in a very narrow range to solve both problems.

In this article we propose a new simple framework, "Partially Acoustic Dark Matter" (PAcDM), that can robustly solve both problems. We assume that DM consists of two components, $\chi_{1}$ and $\chi_{2}$, and that there is also DR that behaves as a tightly coupled relativistic fluid. ${ }^{1}$ The primary component $\chi_{1}$ is cold and collisionless, and dominates the DM mass density. The subdominant component, $\chi_{2}$, is also cold, but is tightly coupled to the DR. The interactions within the DR, and between the DR and $\chi_{2}$, are both strong enough that the tight coupling treatment is valid not only during radiation domination before the CMB epoch but also well into the era of structure formation. Then, since our DR is a tightly coupled relativistic fluid as considered in ref. [23], we can solve the $H_{0}$ problem by choosing the amount of DR appropriately. We will then demonstrate that the persistent $\chi_{2}$-DR interaction inhibits the growth of density perturbations in $\chi_{2}$, which in turn reduces the growth of density fluctuations in the dominant DM component, $\chi_{1}$, provided that the modes in question enter the horizon before matter-radiation equality. The modes at the $8 h^{-1} \mathrm{Mpc}$ scale do indeed come inside the horizon before equality, so we can also solve the $\sigma_{8}$ problem just by choosing an appropriate amount of $\chi_{2}$ to match the observed discrepancy in $\sigma_{8}$.

This class of theories fits very naturally into a "hidden WIMP" scenario [35-38], in which the relic abundance of both DM components $\chi_{1}$ and $\chi_{2}$ is set by annihilation into a hidden sector, rather than into the SM. Massless states in this hidden sector could then constitute interacting DR [39], ameliorating the $H_{0}$ problem, while scattering of $\chi_{2}$ off DR solves the $\sigma_{8}$ problem. In the next section, we construct an explicit model along these lines. However, we stress that the qualitative features of our scenario - an enhancement in $H_{0}$ and a reduction in $\sigma_{8}$ - are robust and only require the coupling constants in the DR- $\chi_{2}$ sector to be sufficiently large that DR is a tightly coupled fluid and $\chi_{2}$ remains in equilibrium with DR. The mechanism is therefore quite general, and is not restricted to a specific DM framework.

The PAcDM paradigm shares some features of the "double-disk dark matter" scenario explored in $[40,41]$, but there are several crucial differences. In particular, since our "dark electrons" are massless, they never form bound states and, consequently, the $\chi_{2}$-DR system never undergoes recombination. Therefore, after matter-radiation equality, it continues to undergo dark acoustic oscillations [42] without being disrupted by "dark recombination." This has the effect of holding back the growth of density perturbations in $\chi_{2}$ until the energy density in DR falls too much for the oscillations to be maintained. Furthermore, by assumption, the $\chi_{2}$-DR system remains tightly coupled throughout the evolution of the universe, even into the era of structure formation. At later times it continues to remain a thermal system, and hence does not virialize. Therefore, we expect that it does not collapse into a disk but instead forms a spherical halo around the galactic center.

\footnotetext{
${ }^{1}$ Earlier work on multi-component DM may be found in, for example, [28-34].
} 
The rest of this paper is organized as follows. In section 2, we describe a concrete, complete model that realizes our solution to the $H_{0}$ and $\sigma_{8}$ problems within the hidden WIMP framework. In section 3 , we develop an analytical understanding of how the $\sigma_{8}$ problem is solved in the PAcDM scenario by studying the linear evolution equations for the density and metric perturbations. This is done without assuming any particular particle physics model of the DM and DR. In section 4, we present the results of our more detailed numerical simulations of the matter power spectrum to determine the precise fraction of $\chi_{2}$ to match the observed discrepancy in $\sigma_{8}$. We then show that the corrections to the CMB spectrum and the CMB lensing measurement are small and within the present uncertainties. We conclude in section 5 .

\section{A benchmark model}

In this section, we construct a benchmark model based on the hidden WIMP paradigm that realizes these ideas. The hidden WIMP framework, in which DM annihilates into hidden sector states rather than into SM particles, has become increasingly attractive as the constraints from direct, indirect, and collider searches continue to get stronger. This class of theories retains the attractive feature of the WIMP paradigm that the DM mass and annihlation cross section are of order the weak scale, a scale that is motivated by a variety of solutions to the hierarchy problem. In the model we consider, both $\chi_{1}$ and $\chi_{2}$ are hidden WIMPs, and their abundances are set by annihilation within the hidden sector.

In a hidden WIMP model the couplings between DM and the SM states may be very small. Nevertheless, as emphasized in ref. [39], if the temperature of the hidden sector is comparable to that of the SM, this class of theories is potentially accessible to experiment. The nature of the signals depends on the masses of the lightest states in the hidden sector. If all the particles in the hidden sector have masses above an $\mathrm{eV}$, these states must decay or annihilate into SM particles before the CMB epoch. This is so as to avoid the over-closure bounds if their masses are above a keV, or the limits on a warm subcomponent of DM if their masses lie between an $\mathrm{eV}$ and a $\mathrm{keV}$. This then implies the existence of couplings between the hidden sector and the SM that can potentially be tested in experiments, as in the exciting DM [35, 43], secluded DM [36], and boosted DM [44, 45] scenarios. If instead the lightest states in the hidden sector have masses below an $\mathrm{eV}$, they are likely to constitute a significant component of the energy density of the universe at the time of matter-radiation equality, potentially leading to observable signals in the CMB. The simplest possibility is that these states, if present, are massless and constitute DR at present times. Provided the hidden sector was in thermal equilibrium with the SM at temperatures at or above the weak scale, there is a lower bound on the contribution of DR to the energy density during the CMB epoch, $\Delta N_{\text {eff }} \gtrsim 0.027$ [39], as expressed in units of the energy density in a single SM neutrino species. This is potentially large enough to be detected in CMB Stage-IV experiments [46-49]. We see that the existence of observable DR is a natural feature in a large class of hidden WIMP models.

DR can take two distinct forms; it may free stream like neutrinos, or scatter with a short mean free path like the particles in a tightly coupled fluid. These two cases can be 
experimentally distinguished, because the details of the CMB spectrum depend not just on the total energy density in radiation, but on the fraction that is free streaming [50-52]. This ratio impacts both the amplitudes of the modes, and the locations of the peaks in the CMB spectrum. The effects of scattering DR and free streaming DR on the CMB are therefore different. In the case when DR scatters, a fit to the Planck CMB data admits values of $\Delta N_{\text {eff }}$ large enough to address the $H_{0}$ problem [23]. As we shall see, scattering of $\chi_{2}$ off the DR can address the $\sigma_{8}$ problem. In this way the hidden WIMP framework is well suited to address both the large scale puzzles.

A concrete, renormalizable and radiatively stable field theory model of a hidden WIMP that annihilates into interacting DR was presented and analyzed in ref. [39]. In this theory, the DR consists of one or more flavors of massless Dirac fermions $\hat{\psi}$ that carry charge under a new $\mathrm{U}(1)$ gauge symmetry. The massless gauge boson associated with this symmetry is denoted by $\hat{A}$. Provided that the U(1) gauge coupling is not too small, $\hat{A}$ and $\hat{\psi}$ behave as tightly coupled DR at late times, and can solve the $H_{0}$ problem.

In the model of ref. [39], the DM was composed of a single component, a complex scalar $\chi$, whose relic abundance was governed by the annihilation through a massive vector boson $\hat{Z}$ into the dark fermions $\hat{\psi}$. It is straightforward to extend this model by introducing a second component of DM, so that we now have two complex scalars, $\chi_{1}$ and $\chi_{2}$. The relic abundances of both $\chi_{1}$ and $\chi_{2}$ are now set by annihilation to the $\hat{\psi}$ through the massive vector boson $\hat{Z}$. A standard thermal freeze-out calculation then tells us that, in such a scenario, the ratio of their relic abundances is given by

$$
\frac{\Omega_{2}}{\Omega_{1}} \sim\left(\frac{m_{2} q_{1}}{m_{1} q_{2}}\right)^{2},
$$

where $m_{1,2}$ and $q_{1,2}$ are the mass and $\hat{Z}$ charge of $\chi_{1,2}$, respectively. We will see that a value of this ratio of order a few $\%$ is required to match the observed $\sim 10 \%$ discrepancy in $\sigma_{8}$. As is clear from the above expression, DM masses and $\hat{Z}$ charges that differ by just a factor of a few can easily result in such a value. The SM and dark sectors can be arranged to be in thermal equilibrium at early times through small Higgs portal couplings $\left|\chi_{1,2}\right|^{2}|H|^{2}$, ensuring that their temperatures are not very different at later times. This model shows that the thermal WIMP framework can provide a simple realization of our solution to the $H_{0}$ and $\sigma_{8}$ problems, without the need to introduce new scales or small parameters.

We now estimate the range of parameters for which this model gives rise to the desired dynamics. First, to solve the $\sigma_{8}$ problem, we want $\chi_{2}$ to be in thermal equilibrium with DR until late times, including the era of structure formation. This requires the momentum transfer rate between the two systems to be much larger than the Hubble rate $H$ until late times. To be quantitative, we define the momentum transfer rate $\Gamma$ from DR to $\chi_{2}$ per degree of freedom of $\chi_{2}$ as

$$
\Gamma \equiv \frac{1}{\left\langle p_{2}^{2}\right\rangle} \frac{\mathrm{d}\left\langle\delta p_{2}^{2}\right\rangle}{\mathrm{d} t},
$$

where $\delta \vec{p}_{2}$ is the change of momentum of $\chi_{2}$ due to a single scattering event with a degree of freedom in DR, and the \langle\rangle indicates thermal average with DR being treated as the bath. Here $t$ is simply the Minkowski time, because $\Gamma$ is a microscopic quantity independent of the 
cosmological expansion, and can be determined in the flat spacetime limit. An elementary calculation shows $\Gamma \propto \hat{\alpha}^{2} \ln \left(\hat{\alpha}^{-1}\right) \hat{T}^{2} / m_{\chi_{2}}$ [24], where $\hat{\alpha}$ is the dark fine structure constant and $\hat{T}$ is the temperature of the dark sector. The proportionality factor is $\mathcal{O}(1)$ and not important, provided that all the dark charges involved in the scattering are $\mathcal{O}(1)$. On the other hand, the Hubble rate $H$ scales as $T^{2}$ during the radiation dominated era, but as $T^{3 / 2}$ during the matter dominated era. Since we want $\Gamma \gg H$ at all times, it follows that the strongest bound comes from the latest times. Demanding $\Gamma \gg H_{0}$ then leads to

$$
\hat{\alpha}^{2} \ln \left(\hat{\alpha}^{-1}\right) \frac{10 \mathrm{GeV}}{m_{\chi_{2}}} \gg 10^{-16}\left(\frac{T_{0}}{\hat{T}}\right)^{2},
$$

where $T_{0}$ is the photon temperature today. It follows that we can easily maintain the thermal equilibrium between $\chi_{2}$ and DR at all times even with fairly small values of the dark gauge coupling. We also need to maintain the equilibrium within DR itself, but this clearly leads to a much weaker condition than the above because the relevant momentum transfer rate simply scales as $\hat{\alpha}^{2} \hat{T}$, without the additional $\hat{T} / m_{\chi_{2}}$ suppression. Finally, note that eq. (2.1) is only valid when the relic abundances of $\chi_{1,2}$ are set by the $\hat{Z}$ gauge coupling, and not by $\hat{\alpha}$. This leads to the condition $\hat{\alpha} \ll \hat{\alpha}_{\hat{Z}}\left(m_{\chi_{1,2}} / m_{\hat{Z}}\right)^{2}$. (If $\hat{\alpha}$ is larger than this, the annihilation through $\hat{A}$ dominates and eq. (2.1) would have to be modified accordingly.) With a typical size of $\hat{\alpha}_{\hat{Z}}$ for WIMPs, we see that this leaves a wide range of parameters for which the $\sigma_{8}$ problems is solved.

Second, in order to solve the $H_{0}$ problem, we require $\Delta N_{\text {eff }}^{\text {scatt }} \gtrsim 0.4[7,9]$, where $\Delta N_{\text {eff }}^{\text {scatt }}$ is the energy density in the scattering component of DR, measured in units of the energy density in a single SM neutrino species. The size of $\Delta N_{\mathrm{eff}}^{\text {scatt }}$ in the concrete model being discussed in this section can be read off from ref. [39]. For example, if the SM and dark sectors kinetically decouple from each other between the QCD phase transition temperature and the $c$-quark mass (which can easily be arranged to be the case), we get $\Delta N_{\text {eff }}^{\text {scatt }} \simeq 0.25$ (or 0.42) for one (or two) flavor(s) of $\hat{\psi}$ and $\hat{T} \simeq 0.39 T_{0}$. These values of $\Delta N_{\mathrm{eff}}^{\mathrm{scatt}}$ are allowed by the current CMB constraints ref. [23, 25, 53], allowing a straightforward solution of the $H_{0}$ problem.

\section{General evolution of DM density perturbations}

In this section, we demonstrate how the $\sigma_{8}$ problem is robustly solved in our framework by performing a simple analysis of the perturbed Einstein equations. More detailed, numerical studies will be presented in the next section. Our analysis here is very general, and essentially depends on only the following assumptions:

- There is a significant contribution to the energy density of the universe from DR around the time of matter-radiation equality. This DR is tightly self-coupled not only during the CMB epoch but also at late times when large scale structure forms.

- DM is cold and comes in two types, a dominant component $\chi_{1}$ that is collisionless, and a subdominant component $\chi_{2}$ that interacts strongly with the DR, again until late times. 
To study how the growth of the perturbations of $\chi_{1}$ is reduced by the scattering of $\chi_{2}$ with DR, we employ the general formalism of Ma and Bertschinger [54] for scalar perturbations. (The study of tensor perturbations is beyond the scope of the present work.) We work in the conformal Newtonian gauge,

$$
\mathrm{d} s^{2}=a^{2}(\tau)\left[-(1+2 \psi) \mathrm{d} \tau^{2}+(1-2 \phi) \delta_{i j} \mathrm{~d} x^{i} \mathrm{~d} x^{j}\right],
$$

where the fields $\psi$ and $\phi$ describe scalar perturbations on the metric. They are determined by four scalar quantities associated with the perturbed energy-momentum tensor $\delta T_{\mu}^{\nu}$, namely, $\delta \equiv \delta \rho / \bar{\rho}=-\delta T_{0}^{0} / \bar{\rho}, \delta P=\delta T_{i}^{i} / 3, \theta \equiv-\partial_{i} \delta T_{0}^{i} /(\bar{\rho}+\bar{P})$, and $\sigma \equiv-\hat{\partial}_{i} \hat{\partial}^{j}\left(\delta T_{j}^{i}-\right.$ $\left.\delta P \delta_{j}^{i}\right) /(\bar{\rho}+\bar{P})$, where $\bar{\rho}$ and $\bar{P}$ are the unperturbed total energy density and pressure, and $\hat{\partial}^{i} \equiv \hat{\partial}_{i} \equiv \partial_{i} / \sqrt{\partial_{j} \partial_{j}}$. For each particle species $s$, we define $\delta_{s} \equiv \delta \rho_{s} / \bar{\rho}_{s}, \theta_{s} \equiv-\partial_{i} \delta T_{s 0}^{i} /\left(\bar{\rho}_{s}+\right.$ $\left.\bar{P}_{s}\right)$, etc. We can also re-express $\theta_{s}$ as the divergence of comoving 3-velocity, $\theta_{s}=\partial_{i} v_{s}^{i}$, where $v^{i} \equiv \mathrm{d} x^{i} / \mathrm{d} \tau . \quad \sigma_{s}$ is the shear stress of the fluid component $s$ and vanishes if $s$ is a perfect fluid. For each $s$ we assume an equation of state of the form $P_{s}=w_{s} \rho_{s}$ with constant $w_{s}$, so the pressures and energy densities are not independent quantities. The total $\delta$ and $\theta$ are given in terms of the individual $\delta_{s}$ and $\theta_{s}$ as $\delta=\sum_{s} \bar{\rho}_{s} \delta_{s} / \bar{\rho}$ and $\theta=\sum_{s}\left(\bar{\rho}_{s}+\bar{P}_{s}\right) \theta_{s} /(\bar{\rho}+\bar{P}) . \sigma$ is related to the individual $\sigma_{s}$ by a sum rule analogous to that for $\theta$. If the total $\sigma$ is zero, the two metric perturbations become equal, $\psi=\phi$. An important parameter in our framework is the ratio $r$ defined as

$$
r \equiv \frac{\bar{\rho}_{2}}{\bar{\rho}_{\mathrm{DM}}}
$$

with $\bar{\rho}_{\mathrm{DM}} \equiv \bar{\rho}_{1}+\bar{\rho}_{2}$.

To linear order in the perturbations, the evolution of the dominant, collisionless component of DM, $\chi_{1}$, is described by

$$
\dot{\delta}_{1}=-\theta_{1}+3 \dot{\phi}, \quad \dot{\theta}_{1}=-\frac{\dot{a}}{a} \theta_{1}+k^{2} \psi,
$$

where the dots indicate derivatives with respect to $\tau$, and we have switched to Fourier space via the transformation $\mathrm{e}^{\mathrm{i} \vec{k} \cdot \vec{x}}$. These equations are just the expressions of local conservation of energy and momentum of $\chi_{1}$ in an expanding FRW background. Being collisionless and cold, $\chi_{1}$ evolves exactly as standard cold DM (CDM) for given $\phi$ and $\psi$. But we will see that in the presence of scattering between $\chi_{2}$ and DR, $\phi$ and $\psi$ are modified away from their values in a standard, single-component CDM model. $\chi_{2}$ evolves as

$$
\dot{\delta}_{2}=-\theta_{2}+3 \dot{\phi}, \quad \dot{\theta}_{2}=-\frac{\dot{a}}{a} \theta_{2}+k^{2} \psi+a \Gamma\left(\theta_{\mathrm{DR}}-\theta_{2}\right),
$$

where $\Gamma$ is the rate of momentum transfer as defined in (2.2), while DR evolves as

$$
\begin{aligned}
& \dot{\delta}_{\mathrm{DR}}=-\frac{4}{3} \theta_{\mathrm{DR}}+4 \dot{\phi}, \\
& \dot{\theta}_{\mathrm{DR}}=k^{2}\left(\frac{\delta_{\mathrm{DR}}}{4}+\psi\right)+\frac{3}{4} \frac{\bar{\rho}_{2}}{\bar{\rho}_{\mathrm{DR}}} a \Gamma\left(\theta_{2}-\theta_{\mathrm{DR}}\right) .
\end{aligned}
$$

Equations (3.4) and (3.5) also just express local energy-momentum conservation, but now conservation applies only to the total $\chi_{2}$-DR system. Accordingly, it may be observed 
that the $\Gamma$ terms cancel for the total $\theta \propto \bar{\rho}_{2} \theta_{2}+\frac{4}{3} \bar{\rho}_{\mathrm{DR}} \theta_{\mathrm{DR}}$, where we have made use of the sum rule for $\theta$ described earlier. It is implicitly assumed in (3.4) and (3.5) that all the constituents of DR (in our case, dark massless electrons and dark photons) are always sufficiently tightly coupled to each other that DR can be treated as a single fluid without a shear stress.

Now, one of our key assumptions is that the $\chi_{2}$-DR interaction rate, $\Gamma$, is also always large enough that the tight coupling approximation can be applied to (3.4) and (3.5) not only during the radiation dominated era but also throughout the matter dominated era. Then, expanding $\theta_{2}, \psi$, etc., in powers of $1 / \Gamma$ in (3.4) and (3.5), we get $\theta_{2}=\theta_{\mathrm{DR}}$ to leading order. Then, cancelling the $\Gamma$ terms by taking an appropriate linear combination of the $\dot{\theta}_{2}$ and $\dot{\theta}_{\mathrm{DR}}$ equations and then setting $\theta_{2}=\theta_{\mathrm{DR}} \equiv \tilde{\theta}$, we obtain

$$
\dot{\tilde{\theta}}=-\frac{\dot{a}}{a} \frac{f}{1+f} \tilde{\theta}+\frac{k^{2}}{4(1+f)} \delta_{\mathrm{DR}}+k^{2} \psi
$$

where $f \equiv 3 \bar{\rho}_{2} / 4 \bar{\rho}_{\mathrm{DR}}$. As it must, this reduces to the equation for $\dot{\theta}_{2}$ or $\dot{\theta}_{\mathrm{DR}}$ without the scattering term in the $f \rightarrow \infty$ (no DR) or $f \rightarrow 0$ (no $\chi_{2}$ ) limits, respectively. Since $f$ grows linearly in $a$, it will eventually be $\gg 1$ and the linearized evolution equations for $\chi_{2}$ will become identical to those for $\chi_{1}$. It follows that, in order to significantly modify the evolution of $\chi_{2}$, it is necessary to have $f \lesssim 1$ when the mode enters the horizon. Recalling the parametrization (3.2) and that $\Delta N_{\mathrm{eff}}=\bar{\rho}_{\mathrm{DR}} / \bar{\rho}_{\nu}$, the value of $f$ at matter-radiation equality is given by

$$
\begin{aligned}
f_{\mathrm{eq}}=\left.\frac{3 \bar{\rho}_{2}}{4 \bar{\rho}_{\mathrm{DR}}}\right|_{\mathrm{eq}} & =\left.\frac{3}{4} \frac{r}{\Delta N_{\mathrm{eff}}} \frac{\bar{\rho}_{\mathrm{DM}}}{\bar{\rho}_{\nu}}\right|_{\mathrm{eq}} \\
& \simeq \frac{3}{4} \frac{r}{\Delta N_{\mathrm{eff}}} \frac{3}{1+\Omega_{\mathrm{B}} / \Omega_{\mathrm{DM}}}\left(1+\left.\frac{\bar{\rho}_{\gamma}}{3 \bar{\rho}_{\nu}}\right|_{\mathrm{eq}}\right),
\end{aligned}
$$

where we have approximated $\left.\bar{\rho}_{\mathrm{DM}}\right|_{\text {eq }}$ as $\bar{\rho}_{\gamma}+\left.3 \bar{\rho}_{\nu}\right|_{\text {eq }}$ since we know that the amount of extra radiation, $\Delta N_{\text {eff }}$, is constrained to be small. Using $\Omega_{\mathrm{B}} / \Omega_{\mathrm{DM}}=0.19$, the above expression gives

$$
f_{\text {eq }} \simeq 0.2 \cdot \frac{r}{0.02} \cdot \frac{0.5}{\Delta N_{\text {eff }}},
$$

where $r=0.02$ and $\Delta N_{\text {eff }}=0.5$ are close to the benchmark values we will use later in our numerical study. To see whether $f_{\text {eq }}$ of 0.2 is small enough to qualify as " $f \ll 1$ ", note that it is comparable to the value of $f$ for the standard baryon acoustic oscillation (BAO) case,

$$
f_{\mathrm{eq}}^{\mathrm{BAO}}=\left.\frac{3 \bar{\rho}_{\mathrm{B}}}{4 \bar{\rho}_{\gamma}}\right|_{\text {eq }}=\frac{3}{4} \frac{1}{1+\Omega_{\mathrm{DM}} / \Omega_{\mathrm{B}}}\left(1+\left.\frac{3 \bar{\rho}_{\nu}}{\bar{\rho}_{\gamma}}\right|_{\text {eq }}\right) \simeq 0.2 .
$$

It follows that the initial behavior of the $\chi_{2}$-DR system after matter-radiation equality is just like that of baryon-photon fluid, namely the density perturbations in $\chi_{2}$ undergo acoustic oscillations. In the meantime, the density perturbations in $\chi_{1}$ grow monotonically. Eventually, around recombination or shortly after, $f$ becomes $\gtrsim 1$ and the perturbations in $\chi_{2}$ begin to grow monotonically as well. This is where the analogy with the baryon-photon fluid breaks down, because the $\chi_{2}$-DR system never undergoes recombination. Once $\rho_{\mathrm{DR}}$ 
becomes subdominant to $\rho_{2}$, DR particles do not have enough energy to maintain dark acoustic oscillations. After this the equations for $\chi_{2}$ take the same form as those for collisionless CDM, and therefore its inhomogeneities in the linear regime evolve just like those of $\chi_{1}$. However, because $\chi_{2}$ 's growth was initially held back for a while, we continue to have $\delta_{2} \ll \delta_{1}$. At very late times, the behavior of $\chi_{1}$ and $\chi_{2}$ are expected to differ on small scales, since $\chi_{1}$ virializes while $\chi_{2}$ remains thermal.

Needless to say, the above conclusion applies only to the modes that enter the horizon before matter-radiation equality, and to a lesser extent also to the modes that come in between the equality and recombination. The modes that come in much later enter the horizon when $f \gg 1$, so they evolve just like $\chi_{1}$ from the beginning, leading to $\delta_{2}=\delta_{1}$. This is a robust prediction of our scenario: the modes with $k \ll k_{\mathrm{eq}} \sim 0.01 \mathrm{Mpc}^{-1}$ should see no modifications in the matter power spectrum compared to standard collisionless CDM.

We are now ready to analyze how the suppression of $\delta_{2}$ affects the evolution of $\chi_{1}$ for the modes with $k \gg k_{\text {eq. }}$. At matter-radiation equality, these modes are already well within the horizon. Then, the relevant component of the perturbed Einstein equation,

$$
k^{2} \phi+3 \frac{\dot{a}}{a}\left(\dot{\phi}+\frac{\dot{a}}{a} \psi\right)=-4 \pi G a^{2} \delta \rho
$$

just reduces to the Newtonian, Poisson equation, $k^{2} \phi \simeq-4 \pi G a^{2} \delta \rho$. (The $a^{2}$ on the righthand side is there because the $k$ on the left-hand side is the comoving wave number related to the physical wave number $k_{\text {phys }}$ as $k_{\text {phys }}=k / a$, with $a=1$ today by convention.) Here, recalling the sum rule $\delta \rho=\sum_{s} \bar{\rho}_{s} \delta_{s}$, we have

$$
\begin{aligned}
\delta \rho & =\bar{\rho}_{1} \delta_{1}+\bar{\rho}_{2} \delta_{2} \\
& =\bar{\rho}_{\mathrm{DM}}\left[(1-r) \delta_{1}+r \delta_{2}\right],
\end{aligned}
$$

where for simplicity we have only kept $\chi_{1}$ and $\chi_{2}$, while ignoring the other components such as baryons. (However, these are included in our numerical studies to be discussed later.) If $\chi_{2}$ were also collisionless CDM like $\chi_{1}$, we would have $\delta_{1}=\delta_{2} \equiv \delta_{\mathrm{DM}}$. Then the above relation would just lead to $\delta \rho=\bar{\rho}_{\mathrm{DM}} \delta_{\mathrm{DM}}$, as if we only had a single component of CDM. However, as discussed above, the $\chi_{2}$-DR interactions lead to $\delta_{1} \gg \delta_{2}$ for the modes with $k \gg k_{\text {eq }}$, so we instead have

$$
\delta \rho \simeq(1-r) \bar{\rho}_{\mathrm{DM}} \delta_{1} .
$$

This result is completely intuitive: if $\chi_{2}$ is not fluctuating, the fluctuations in the spacetime metric should only depend on the fluctuations in $\chi_{1}$, but $\chi_{1}$ is only $(1-r)$ of the total mass density. Then, the Poisson equation becomes

$$
\begin{aligned}
k^{2} \phi & \simeq-4 \pi a^{2} G \bar{\rho}_{\mathrm{DM}} \cdot(1-r) \delta_{1} \\
& =-\frac{6}{\tau^{2}} \cdot(1-r) \delta_{1},
\end{aligned}
$$

where we have used the Friedman equation and the fact that $a \propto \tau^{2}$ during matter domination. Defining a dimensionless variable $\eta \equiv k \tau$, the above equation becomes

$$
\phi \simeq-\frac{6(1-r)}{\eta^{2}} \delta_{1} .
$$


On the other hand, cancelling $\theta_{1}$ between the two equations in (3.3), we get

$$
\delta_{1}^{\prime \prime}+\frac{2}{\eta} \delta_{1}^{\prime}=-\psi+\frac{6}{\eta} \phi^{\prime}+3 \phi^{\prime \prime} .
$$

where the primes denote derivatives with respect to $\eta$. Since the modes in question are well within the horizon, we have $\eta \gg 1$ by definition and the $\phi^{\prime}$ and $\phi^{\prime \prime}$ terms can be neglected compared to the $\psi$ term. For simplicity, let us ignore the shear stresses in the photons and neutrinos, since our primary focus is on the difference between the $r=0$ and $r \neq 0$ cases. Then we have $\psi=\phi$, and so combining the above equation with eq. (3.14) leads to

$$
\delta_{1}^{\prime \prime}+\frac{2}{\eta} \delta_{1}^{\prime} \simeq-\phi \simeq \frac{6(1-r)}{\eta^{2}} \delta_{1}
$$

This tells us that $\delta_{1}$ is given by a linear combination of $\eta^{-1 / 2 \pm \sqrt{25 / 4-6 r}}$. The solution with the negative power of $\eta$ is important for matching the "initial" condition at $a=a_{\text {eq }}$, but then it quickly decays away. Then the late-time behavior of the modes of $\chi_{1}$ with $k \gg k_{\text {eq }}$ is approximately given by

$$
\delta_{1} \propto\left(\frac{\eta}{\eta_{\mathrm{eq}}}\right)^{-1 / 2+\sqrt{25 / 4-6 r}} \propto\left(\frac{a}{a_{\mathrm{eq}}}\right)^{1-0.6 r+\mathcal{O}\left(r^{2}\right)} .
$$

Taking the ratio of our case $(r>0)$ to standard, single-component, collisionless CDM $(r=0)$, we obtain

$$
\frac{\delta_{1}(r)}{\delta_{1}(0)}=\left(\frac{a}{a_{\mathrm{eq}}}\right)^{-0.6 r+\mathcal{O}\left(r^{2}\right)},
$$

where we have neglected the mild $r$ dependence in the proportionality factor. This is justified because inflation gives us the same, universal initial conditions for the perturbations, irrespective of $r$ (see eqs. (4.1) and (4.2)), and the subsequent evolution of $\chi_{1}$ during radiation domination is controlled by radiation and hence is only mildly affected by the presence of $\chi_{2}$. We see from eq. (3.18) that $\delta_{1}$ grows slower than in the CDM case. $^{2}$

It follows that, in this approximation, the ratio of our power spectrum to the standard one is given by

$$
\frac{P(r)}{P(0)}=\frac{[\delta \rho(r)]^{2}}{[\delta \rho(0)]^{2}}=\frac{(1-r)^{2}\left[\delta_{1}(r)\right]^{2}}{\left[\delta_{1}(0)\right]^{2}} \simeq(1-2 r)\left(\frac{a}{a_{\mathrm{eq}}}\right)^{-1.2 r},
$$

where we have used the relation (3.12) in the second step and dropped $\mathcal{O}\left(r^{2}\right)$ terms at the end. As discussed already, the above suppression applies only to the modes with $k \gg k_{\text {eq }}$, and the modes with $k \ll k_{\text {eq }}$ see no suppression. The upper and lower panels of figure 1 in section 4 show the results of a numerical analysis that confirms this analytical understanding.

\footnotetext{
${ }^{2}$ Although the underlying dynamics is different, it is possible to get a similar effect by increasing the neutrino masses [55].
} 


\section{Numerical results and corrections to the CMB spectrum}

In this section, we obtain numerical results for the matter power spectrum in PAcDM framework. We also show that the effects of $\chi_{2}$-DR scattering on the CMB spectrum are small and within current experimental uncertainties.

The evolution of the perturbations in $\chi_{1,2}$ and DR is governed by eqs. (3.3)-(3.5) and (3.10), where the dark $\mathrm{U}(1)$ coupling is chosen to satisfy the condition (2.3). The equations for baryons and photons are obtained simply by taking eqs. (3.4) and (3.5) and relabelling $\chi_{2} \rightarrow \mathrm{B}$ (baryon) and $\mathrm{DR} \rightarrow \gamma$, again in the tight coupling limit. The tight coupling approximation means our analysis fails to properly capture the physics of recombination and photon diffusion. We also ignore the effect of neutrinos. These limitations are not an immediate concern for us because we are only interested in how $\chi_{2^{-}}$ DR scattering affects the CMB spectrum compared to the cases without such scattering, with or without DR, and the effects we ignore are common to all cases. Finally, photon polarizations are not distinguished in our treatment, but this is a small effect as we focus only on the matter and CMB temperature power spectra.

The tight coupling approximation and the absence of shear stress from neutrino free streaming imply that $\phi=\psi$. Assuming inflation, the initial conditions for the perturbations are then simply given by the superhorizon solutions of all the evolution equations above, together with the assumption of adiabatic perturbations that entropy per matter particle is unchanged by perturbations. For the modes that enter the horizon during radiation domination, the initial conditions are given by

$$
\delta_{\gamma, \mathrm{DR}}=\frac{4}{3} \delta_{1,2, \mathrm{~B}}=-2 \psi, \quad \theta_{1,2, \mathrm{DR}, \mathrm{B}, \gamma}=\frac{k^{2} \tau}{2} \psi
$$

while for those that come in during matter domination we have

$$
\frac{3}{4} \delta_{\gamma, \mathrm{DR}}=\delta_{1,2, \mathrm{~B}}=-2 \psi, \quad \theta_{1,2, \mathrm{DR}, \mathrm{B}, \gamma}=\frac{k^{2} \tau}{3} \psi
$$

We neglect the tilt in the primordial spectrum (i.e, $n_{s}=1$ ) and use a $k$-independent value $10^{-4}$ for the initial perturbation $-2 \psi$ above, but the precise choice of this number is immaterial as we will only compare the ratio of the spectrum with $r \neq 0$ to that with $r=0$.

We are now ready to evolve the perturbations numerically as governed by the equations and initial conditions described above, We choose the values $h=0.68, \Omega_{\gamma} h^{2}=2.47 \times 10^{-5}$, $\Omega_{\Lambda} h^{2}=0.69, \Omega_{b} h^{2}=2.2 \times 10^{-2}$ and $\Omega_{\nu}=0.69 \Omega_{\gamma}[4]$. $\Omega_{\Lambda}$ only has small effects on our results so its precise value is not important for our purpose here. We choose $\Delta N_{\text {eff }}^{\text {scatt }}=$ 0.4 and a slightly larger value of $\Omega_{\mathrm{DM}} h^{2}=0.13$ in order to keep the redshift at matterradiation equality unchanged. This allows us to compare our matter power spectrum to that of a conventional single component DM model without any DR. We find the choice of $r=2.0 \%$ leads to a $10 \%$ suppression in the matter power spectrum around the scale $k \sim 0.2 h \mathrm{Mpc}^{-1}$ compared to $\Lambda \mathrm{CDM}$, thereby solving the $\sigma_{8}$ problem. It should be noted that this corresponds to a suppression of about $20 \%$ compared to the $r=0$ case with 
the same amount of DR, as shown in figure 1, and confirms the features we identified analytically in section $3 .^{3}$

The lower panel of figure 1 shows the results with the same ratios $r$ of DM components, but with a reduced amount of DR, $\Delta N_{\text {eff }}^{\text {scatt }}=0.05$. Notice that the suppression in $\sigma_{8}$ is nevertheless almost identical to the case with $\Delta N_{\text {eff }}^{\text {scatt }}=0.4$. The essential reason why the reduction in $\sigma_{8}$ is so robust is that, even with $\Delta N_{\text {eff }}^{\text {scatt }}$ as small as 0.05 , there remains enough DR for $\chi_{2}$ to scatter with at the time when the $\sigma_{8}$ modes enter the horizon, which is well before matter-radiation equality. Therefore, our mechanism would still constitute a solution to the $\sigma_{8}$ problem even if future measurements were to settle on a smaller $\Delta N_{\text {eff }}^{\text {scatt }}$.

Let us now discuss the impact of $\chi_{2}$-DR interactions on the CMB spectrum. We compare the temperature power spectra of the $r=2.0 \%$ and $r=0$ cases with the same amount of DR. Since our equations do not include the physics of recombination or photon diffusion, we halt the evolution just before recombination at $a=10^{-3}$ (which is when the electron number density begins to fall exponentially) and then evaluate the following quantity:

$$
\left(\frac{\delta T}{T}\right)_{*} \equiv \frac{1}{4} \delta_{\gamma}+\psi
$$

This has the same form as the standard expression for $\delta T / T$ for the CMB, except for the absence of small Doppler effect corrections [57] and the fact that it is evaluated right before recombination as opposed to the time of photon decoupling. Nevertheless, we map $(\delta T / T)_{*}$ to the $C_{\ell}$ coefficients as if $(\delta T / T)_{*}$ were $\delta T / T$. In other words, we treat the system as if the photons instantaneously decouple right before recombination, and obtain a "snapshot" of the CMB spectrum at that time. This is sufficient for the purpose of showing that the effects of $\chi_{2}$-DR interactions have very small impact on the CMB spectrum, because the effects of such interactions on the photons at and after recombination are small. Moreover, there is very little time between recombination and photon decoupling. Hence, if the CMB spectrum with $r=2.0 \%$ is very similar to that with $r=0$ right before recombination, we expect that they should continue to remain similar afterwards. In figure 2, we compare the primordial spectra of the $r \neq 0$ (red solid) and $r=0$ (black solid) cases. The $\chi_{2}$-DR scattering damps the CMB spectrum, but the deviations are always less than $2 \%$, which is within the uncertainties of current measurements. The largest deviations are for modes in the range $700 \lesssim \ell \lesssim 1600$. The reason that the modes with $\ell \lesssim 700$ see very little deviation is because they enter the horizon late and by that time the DR has become too cold to affect the physics, as discussed in section 3. On the other hand, the modes with $\ell \gtrsim 1600$ enter the horizon at a time when the energy density is completely dominated by radiation and so the metric perturbations damp quickly, resulting in small corrections to $\delta_{\gamma}$.

The other aspect of the last point above is that the metric perturbations are appreciably modified only after matter-radiation equality. This means that the observations of CMB lensing effects can porentially constrain our scenario. The key quantity for calculating the impact of lensing on the temperature power spectrum is the lensing power spectrum $C_{\ell}^{\phi \phi}$ as

\footnotetext{
${ }^{3}$ The detailed CMB and Large Scale Structure constraints on Partially-Interacting DM were studied in [42] using the Planck 2013 data [56]. The bound allows $\lesssim 5 \%$ of the DM density to be tightly coupled to DR. Since we require an $r$ of order $2 \%$, our result is consistent with this limit.
} 

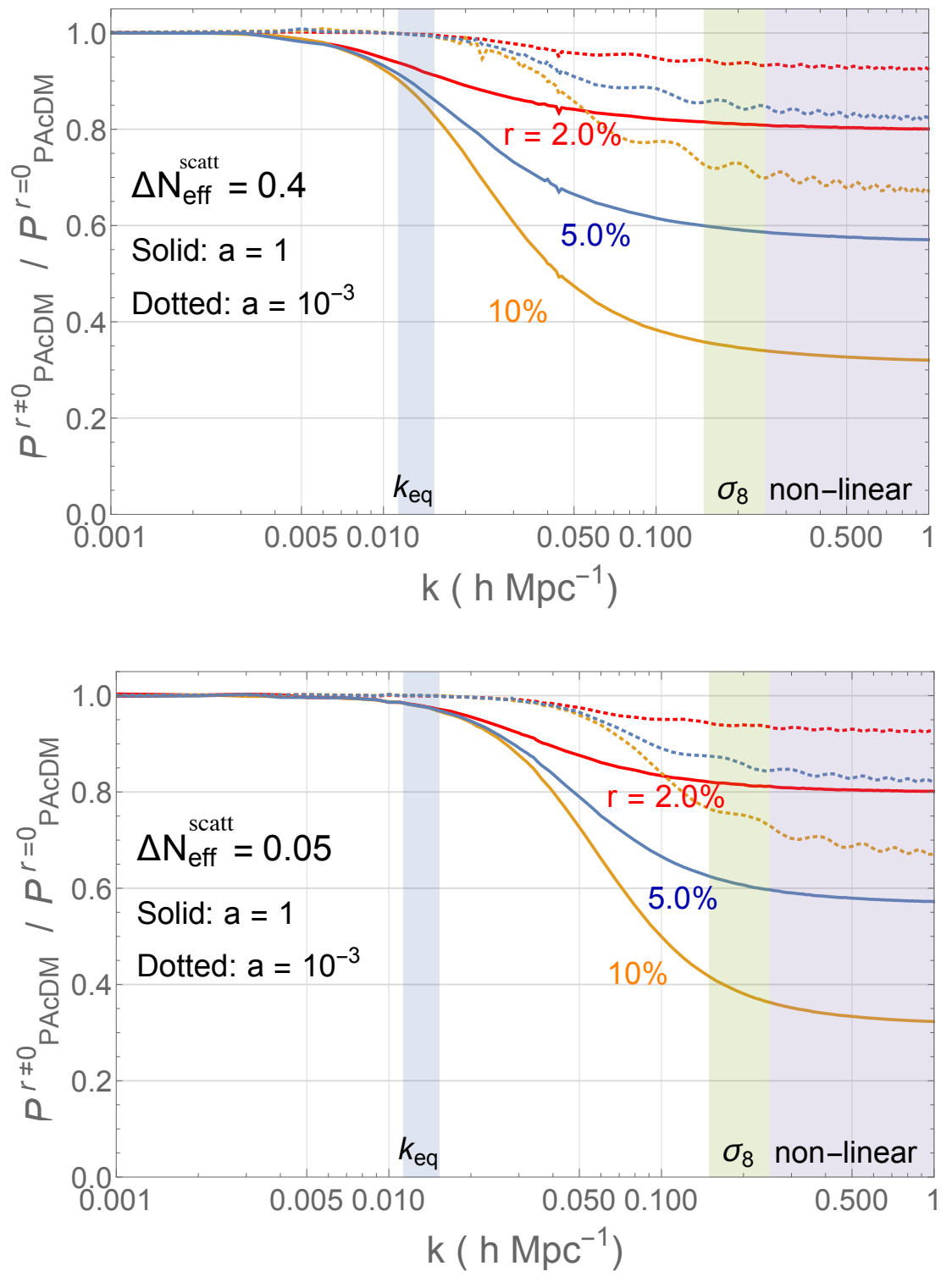

Figure 1. Upper: ratio of the DM power spectrum of the $r \neq 0$ case to the $r=0$ case, both with $\Delta N_{\text {eff }}^{\text {scatt }}=0.4$. The curves are obtained by numerically solving the linear evolution equations (3.3)-(3.5) and the perturbed Einstein equation (3.10), all in the tight coupling limit and assuming no anisotropic stress (hence $\sigma=0$ and $\phi=\psi$ ). Results for different values of $r$ are labelled in different colors, while earlier $\left(a=10^{-3}\right)$ and later $(a=1)$ times are indicated by dotted and solid lines, respectively. For the smaller scale structures $k \gtrsim 0.2 h \mathrm{Mpc}^{-1}$, nonlinear gravitational effects become important so our linear approximation is no longer reliable. Lower: same plot but with a reduced amount of DR, $\Delta N_{\mathrm{eff}}^{\text {scatt }}=0.05$. 


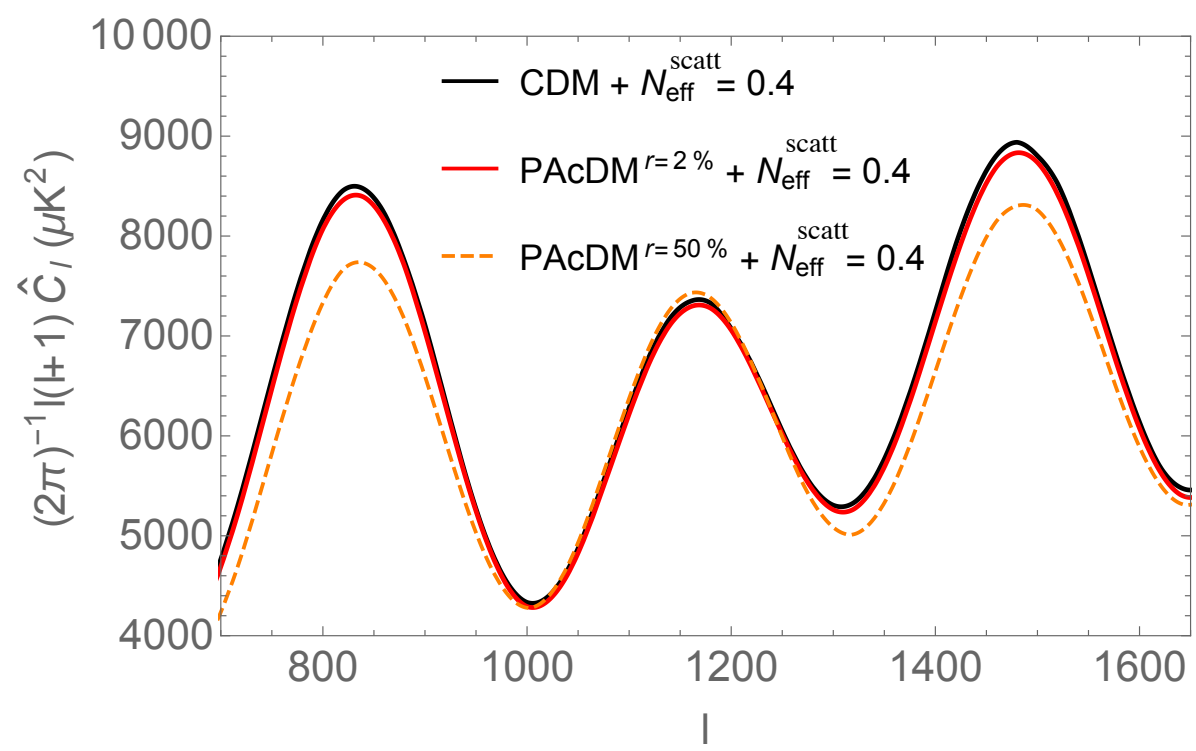

Figure 2. A comparison of the CMB spectrum between PAcDM and CDM models, assuming $\Delta N_{\mathrm{eff}}^{\mathrm{scatt}}=0.4$ in both cases. The black (red) curve is for the $\Lambda$ CDM (PAcDM) model, derived from the $\left\{\delta_{\gamma}, \theta_{\gamma}\right\}$ result in the linear evolution equations. The PAcDM model assumes the DM ratio $r=2.0 \%$. For comparison, we also show a PAcDM model with $r=50 \%$, which exhibits a clear enhancement of the expansion peaks and suppression of the compression peaks due to the pressure of tightly coupled DR.

a function of multipole $\ell$. Using the Limber approximation [58], we estimate the spectrum using [59]

$$
\ell^{4} C_{\ell}^{\phi \phi} \propto \int_{0}^{\chi *} \mathrm{~d} \chi\left(1-\chi / \chi_{*}\right)^{2} k \phi^{2}(k, a) g^{2}(\chi)
$$

where $\chi$ is the comoving distance from the observer and hence $k=\ell / \chi$. In this expression the scale factor $a$ is to be evaluated at $\chi$, i.e., $a=a(\chi)$. The parameter $\chi_{*} \simeq 10^{4} \mathrm{Mpc}$ corresponds to the value of $\chi$ of the last scattering surface. The lensing effect is captured by $\left(1-\chi / \chi_{*}\right)^{2}$. The function $g(\chi)$ effectively describes the decays of metric perturbations due to the vacuum energy at late times. Its value is therefore equal to 1 by definition during matter domination $\left(\chi \gtrsim 0.5 \chi_{*}\right)$ and starts to decrease once $\chi$ drops below $\simeq 0.5 \chi_{*}$. The transfer function is already folded into the above expression, so $\phi(k)$ is simply the primordial metric fluctuation determined by the linear perturbation equations discussed in section 3 .

In the Planck data [14], the smallest percentage error in $C_{\ell}^{\phi \phi}$ is $\simeq 5 \%$ at $\ell \simeq 150$. The $C_{\ell}^{\phi \phi}$ at this multipole gets its main contributions from $\chi \simeq 0.4 \chi_{*}$ in eq. (4.4), which corresponds to the modes with $k \simeq \mathcal{O}(0.01) \mathrm{Mpc}^{-1}$. The deviation in $\phi^{2}$ between the $r=2.0 \%$ and $r=0$ cases is $\simeq 4 \%$ for this value of $k$ (see figure 1 ), and the integral (4.4) gives a $2.5 \%$ deviation between the two cases. This is within the current uncertainties. 


\section{Conclusions}

We have presented a new framework in which DM is composed of two distinct components that can provide a solution to both the $H_{0}$ and $\sigma_{8}$ problems. While the dominant component of DM is cold and collisionless, the subdominant component is also cold but interacts strongly with DR, which itself constitutes a tightly coupled fluid. Our framework is very general and can be adopted in a wide variety of DM models. In particular, it can easily be accommodated in the hidden WIMP framework, with both constituents of DM arising as thermal relics. Our scenario predicts distinctive modifications to the matter and CMB power spectra, allowing it to be tested by future experiments.

By solving a set of linear evolution equations, we have shown that the observed $10 \%$ discrepancy in $\sigma_{8}$ requires the mass density in the subdominant, interacting DM species to constitute $\simeq 2.0 \%$ of the total DM density, while the amount of DR can be separately chosen to fix the $H_{0}$ problem. This apparently small ratio of the two DM components could easily arise in, for example, the WIMP framework without introducing hierarchically small parameters into the Lagrangian. The required tight couplings between the interacting DM and DR and within DR itself can be obtained in a wide range of perturbative coupling constants, as we showed in a concrete model. We found that, with an interacting DM component of about $2 \%$ to solve the $\sigma_{8}$ problem and the appropriate amount of DR to address the $H_{0}$ problem, the deviations in the $\mathrm{CMB}$ spectrum and the CMB lensing measurements are well within the current uncertainties.

It is interesting to compare and contrast our proposal with the scenario put forward in [24-26]. In the PAcDM framework, only a subcomponent of DM experiences acoustic oscillations, while the primary component of DM is responsible for building up structure. As seen in eq. (3.19), this suppresses the rate of growth of power during the era of matter domination, with the result that most of the corrections to the DM density perturbations, and hence corrections to the gravitational potential, arise well after matter-radiation equality. However, in the proposal of [24-26], the entirety of DM undergoes oscillations prior to matter-radiation equality that continue through to the CMB epoch. In this case, the corrections to the DM density perturbations are already significant at the time of matterradiation equality, and the resulting corrections to the CMB are expected to be significantly larger. Hence future precision studies of the CMB may be able to distinguish these two classes of models.

It is important to note that our mechanism to reduce $\sigma_{8}$ is not especially sensitive to the precise value of $\Delta N_{\text {eff }}^{\text {scatt }}$. Hence, if future measurements were to settle on a smaller $\Delta N_{\text {eff }}^{\text {scatt }}$, our mechanism would still constitute a solution to the $\sigma_{8}$ problem. Note that lowering $\Delta N_{\text {eff }}^{\text {scatt }}$ would also imply even smaller corrections to the CMB, since most of the modes that are observed in the CMB would now enter the horizon at a time when the contribution of DR to the energy density is small.

While the primary focus of this article is on large scale structure, our framework may also have potentially observable effects on smaller scales. It follows from eq. (2.3) that at each locale in the universe the $\chi_{2}$ particles continue to experience a sufficient number of collisions with surrounding DR particles to maintain thermal equilibrium, at least locally, 
at all times. This remains true during the era of structure formation. The same condition also ensures that the DR itself remains a tightly coupled relativistic fluid at all times. Being strictly massless, the dark charged particles in DR never undergo recombination. Being tightly coupled, DR is non-dissipative and hence behaves as a perfect thermal fluid. Because of these properties, which are qualitatively different from those of the baryonphoton system, we expect that the $\chi_{2}$-DR system does not collapse into a disk but instead forms a smooth, spherical halo around the galactic center. This is qualitatively distinct from the dynamics of the recently proposed "double-disk DM" or partially dissipative DM [40]. The existence of this halo would impact galactic dynamics, with the exact nature of its effects depending on the details of its density profile. We defer a careful study of these effects on the small scale structure of DM halos for future work.

The current discordance between direct and indirect measurements of $H_{0}$ and $\sigma_{8}$ may be the first hint for new cosmology beyond the $\Lambda \mathrm{CDM}$ paradigm. These discrepancies can be naturally addressed within the non-minimal dark sector structure we have proposed. In the coming years, the experimental precision in the indirect measurement of $N_{\text {eff }}$ and $\sigma_{8}$ from the CMB [46-49] (e.g. CMB stage-IV), and in direct measurements of the Hubble constant [60,61], and $\sigma_{8}[49,62]$ (LSST, DESI) are all expected to improve significantly. If the current discrepancies in the $H_{0}$ and $\sigma_{8}$ measurements are indeed due to new physics, these future experiments have great potential for distinguishing between different candidate theories such as the framework presented in this paper.

\section{Acknowledgments}

We thank Gustavo Marques-Tavares, Valentina Prilepina, Matthew Reece, Martin Schmaltz, Neelima Sehgal and Yong Tang for helpful discussions. ZC, SH and YT are supported in part by the National Science Foundation under grant PHY-1315155, and by the Maryland Center for Fundamental Physics. SH is also supported in part by a fellowship from The Kwanjeong Educational Foundation. YC is supported by Perimeter Institute for Theoretical Physics, which is supported by the Government of Canada through Industry Canada and by the Province of Ontario through the Ministry of Research and Innovation. YC is also supported in part by the Maryland Center for Fundamental Physics. TO is supported by the US Department of Energy under grant DE-SC0010102. YC, TO, and YT all thank the Aspen Center for Physics, which is supported by National Science Foundation grant PHY-1066293.

Open Access. This article is distributed under the terms of the Creative Commons Attribution License (CC-BY 4.0), which permits any use, distribution and reproduction in any medium, provided the original author(s) and source are credited.

\section{References}

[1] B. Moore, Evidence against dissipationless dark matter from observations of galaxy haloes, Nature 370 (1994) 629 [INSPIRE]. 
[2] R.A. Flores and J.R. Primack, Observational and theoretical constraints on singular dark matter halos, Astrophys. J. 427 (1994) L1 [astro-ph/9402004] [INSPIRE].

[3] M. Boylan-Kolchin, J.S. Bullock and M. Kaplinghat, Too big to fail? The puzzling darkness of massive Milky Way subhaloes, Mon. Not. Roy. Astron. Soc. 415 (2011) L40 [arXiv: 1103.0007] [INSPIRE].

[4] Planck collaboration, P.A.R. Ade et al., Planck 2015 results. XIII. Cosmological parameters, Astron. Astrophys. 594 (2016) A13 [arXiv:1502.01589] [INSPIRE].

[5] A.G. Riess et al., A 3\% Solution: Determination of the Hubble Constant with the Hubble Space Telescope and Wide Field Camera 3, Astrophys. J. 730 (2011) 119 [Erratum ibid. 732 (2011) 129] [arXiv:1103.2976] [INSPIRE].

[6] S.H. Suyu et al., Two accurate time-delay distances from strong lensing: Implications for cosmology, Astrophys. J. 766 (2013) 70 [arXiv:1208.6010] [INSPIRE].

[7] A.G. Riess et al., A 2.4\% Determination of the Local Value of the Hubble Constant, Astrophys. J. 826 (2016) 56 [arXiv:1604.01424] [INSPIRE].

[8] E. Di Valentino, A. Melchiorri and J. Silk, Reconciling Planck with the local value of $H_{0}$ in extended parameter space, Phys. Lett. B 761 (2016) 242 [arXiv:1606.00634] [INSPIRE].

[9] J.L. Bernal, L. Verde and A.G. Riess, The trouble with $H_{0}$, JCAP 10 (2016) 019 [arXiv: 1607.05617] [INSPIRE].

[10] C. Heymans et al., CFHTLenS tomographic weak lensing cosmological parameter constraints: Mitigating the impact of intrinsic galaxy alignments, Mon. Not. Roy. Astron. Soc. 432 (2013) 2433 [arXiv: 1303.1808] [inSPIRE].

[11] Planck collaboration, P.A.R. Ade et al., Planck 2013 results. XX. Cosmology from Sunyaev-Zeldovich cluster counts, Astron. Astrophys. 571 (2014) A20 [arXiv:1303.5080] [INSPIRE].

[12] N. MacCrann, J. Zuntz, S. Bridle, B. Jain and M.R. Becker, Cosmic Discordance: Are Planck CMB and CFHTLenS weak lensing measurements out of tune?, Mon. Not. Roy. Astron. Soc. 451 (2015) 2877 [arXiv: 1408.4742] [INSPIRE].

[13] L. Fu et al., CFHTLenS: Cosmological constraints from a combination of cosmic shear two-point and three-point correlations, Mon. Not. Roy. Astron. Soc. 441 (2014) 2725 [arXiv: 1404.5469] [INSPIRE].

[14] Planck collaboration, P.A.R. Ade et al., Planck 2015 results. XV. Gravitational lensing, Astron. Astrophys. 594 (2016) A15 [arXiv:1502.01591] [INSPIRE].

[15] C.L. Reichardt et al., A measurement of secondary cosmic microwave background anisotropies with two years of South Pole Telescope observations, Astrophys. J. 755 (2012) 70 [arXiv: 1111.0932] [INSPIRE].

[16] M. Hasselfield et al., The Atacama Cosmology Telescope: Sunyaev-Zel'dovich selected galaxyclusters at $148 \mathrm{GHz}$ from three seasons of data, JCAP 07 (2013) 008 [arXiv: 1301.0816] [INSPIRE].

[17] Planck collaboration, P.A.R. Ade et al., Planck 2015 results. XXIV. Cosmology from Sunyaev-Zeldovich cluster counts, Astron. Astrophys. 594 (2016) A24 [arXiv:1502.01597] [INSPIRE]. 
[18] S. Joudaki et al., CFHTLenS revisited: assessing concordance with Planck including astrophysical systematics, arXiv:1601.05786 [INSPIRE].

[19] T.D. Kitching, L. Verde, A.F. Heavens and R. Jimenez, Discrepancies between CFHTLenS cosmic shear and Planck: new physics or systematic effects?, Mon. Not. Roy. Astron. Soc. 459 (2016) 971 [arXiv:1602.02960] [INSPIRE].

[20] K. Enqvist, S. Nadathur, T. Sekiguchi and T. Takahashi, Decaying dark matter and the tension in $\sigma_{8}, J C A P 09$ (2015) 067 [arXiv: 1505.05511] [INSPIRE].

[21] V. Poulin, P.D. Serpico and J. Lesgourgues, A fresh look at linear cosmological constraints on a decaying dark matter component, JCAP 08 (2016) 036 [arXiv:1606.02073] [INSPIRE].

[22] R.A. Battye, T. Charnock and A. Moss, Tension between the power spectrum of density perturbations measured on large and small scales, Phys. Rev. D 91 (2015) 103508 [arXiv:1409.2769] [INSPIRE].

[23] D. Baumann, D. Green, J. Meyers and B. Wallisch, Phases of New Physics in the CMB, JCAP 01 (2016) 007 [arXiv: 1508.06342] [INSPIRE].

[24] M.A. Buen-Abad, G. Marques-Tavares and M. Schmaltz, Non-Abelian dark matter and dark radiation, Phys. Rev. D 92 (2015) 023531 [arXiv: 1505.03542] [INSPIRE].

[25] J. Lesgourgues, G. Marques-Tavares and M. Schmaltz, Evidence for dark matter interactions in cosmological precision data?, JCAP 02 (2016) 037 [arXiv: 1507.04351] [INSPIRE].

[26] P. Ko and Y. Tang, Light dark photon and fermionic dark radiation for the Hubble constant and the structure formation, Phys. Lett. B 762 (2016) 462 [arXiv:1608.01083] [INSPIRE].

[27] P. Ko and Y. Tang, Residual Non-Abelian Dark Matter and Dark Radiation, arXiv: 1609.02307 [INSPIRE].

[28] H. Goldberg and L.J. Hall, A New Candidate for Dark Matter, Phys. Lett. B 174 (1986) 151 [INSPIRE].

[29] M. Yu. Khlopov, G.M. Beskin, N.E. Bochkarev, L.A. Pustylnik and S.A. Pustylnik, Observational Physics of Mirror World, Sov. Astron. 35 (1991) 21 [Astron. Zh. 68 (1991) 42] [INSPIRE].

[30] Z.G. Berezhiani, A.D. Dolgov and R.N. Mohapatra, Asymmetric inflationary reheating and the nature of mirror universe, Phys. Lett. B 375 (1996) 26 [hep-ph/9511221] [INSPIRE].

[31] D.E. Kaplan, G.Z. Krnjaic, K.R. Rehermann and C.M. Wells, Atomic Dark Matter, JCAP 05 (2010) 021 [arXiv:0909.0753] [InSPIRE].

[32] D.E. Kaplan, G.Z. Krnjaic, K.R. Rehermann and C.M. Wells, Dark Atoms: Asymmetry and Direct Detection, JCAP 10 (2011) 011 [arXiv:1105.2073] [InSPIRE].

[33] F.-Y. Cyr-Racine and K. Sigurdson, Cosmology of atomic dark matter, Phys. Rev. D 87 (2013) 103515 [arXiv:1209.5752] [InSPIRE].

[34] K.R. Dienes and B. Thomas, Dynamical Dark Matter: I. Theoretical Overview, Phys. Rev. D 85 (2012) 083523 [arXiv:1106.4546] [INSPIRE].

[35] D.P. Finkbeiner and N. Weiner, Exciting Dark Matter and the INTEGRAL/SPI $511 \mathrm{keV}$ signal, Phys. Rev. D 76 (2007) 083519 [astro-ph/0702587] [INSPIRE].

[36] M. Pospelov, A. Ritz and M.B. Voloshin, Secluded WIMP Dark Matter, Phys. Lett. B 662 (2008) 53 [arXiv:0711.4866] [INSPIRE]. 
[37] J.L. Feng and J. Kumar, The WIMPless Miracle: Dark-Matter Particles without Weak-Scale Masses or Weak Interactions, Phys. Rev. Lett. 101 (2008) 231301 [arXiv:0803.4196] [INSPIRE].

[38] J.L. Feng, H. Tu and H.-B. Yu, Thermal Relics in Hidden Sectors, JCAP 10 (2008) 043 [arXiv:0808.2318] [INSPIRE].

[39] Z. Chacko, Y. Cui, S. Hong and T. Okui, Hidden dark matter sector, dark radiation and the CMB, Phys. Rev. D 92 (2015) 055033 [arXiv:1505.04192] [INSPIRE].

[40] J. Fan, A. Katz, L. Randall and M. Reece, Double-Disk Dark Matter, Phys. Dark Univ. 2 (2013) 139 [arXiv: 1303.1521] [INSPIRE].

[41] J. Fan, A. Katz, L. Randall and M. Reece, Dark-Disk Universe, Phys. Rev. Lett. 110 (2013) 211302 [arXiv: 1303.3271] [INSPIRE].

[42] F.-Y. Cyr-Racine, R. de Putter, A. Raccanelli and K. Sigurdson, Constraints on Large-Scale Dark Acoustic Oscillations from Cosmology, Phys. Rev. D 89 (2014) 063517 [arXiv: 1310.3278] [INSPIRE].

[43] I. Cholis, L. Goodenough and N. Weiner, High Energy Positrons and the WMAP Haze from Exciting Dark Matter, Phys. Rev. D 79 (2009) 123505 [arXiv:0802.2922] [INSPIRE].

[44] K. Agashe, Y. Cui, L. Necib and J. Thaler, (In)direct Detection of Boosted Dark Matter, JCAP 10 (2014) 062 [arXiv: 1405.7370] [INSPIRE].

[45] J. Berger, Y. Cui and Y. Zhao, Detecting Boosted Dark Matter from the Sun with Large Volume Neutrino Detectors, JCAP 02 (2015) 005 [arXiv: 1410.2246] [INSPIRE].

[46] K.N. Abazajian et al., Neutrino Physics from the Cosmic Microwave Background and Large Scale Structure, Astropart. Phys. 63 (2015) 66 [arXiv:1309.5383] [InSPIRE].

[47] J. Errard, S.M. Feeney, H.V. Peiris and A.H. Jaffe, Robust forecasts on fundamental physics from the foreground-obscured, gravitationally-lensed CMB polarization, JCAP 03 (2016) 052 [arXiv: 1509.06770] [INSPIRE].

[48] W.L.K. Wu, J. Errard, C. Dvorkin et al., A Guide to Designing Future Ground-based Cosmic Microwave Background Experiments, Astrophys. J. 788 (2014) 138 [arXiv:1402.4108] [INSPIRE].

[49] S. Dodelson, K. Heitmann, C. Hirata et al., Cosmic Visions Dark Energy: Science, arXiv:1604.07626 [INSPIRE].

[50] P. Peebles, The role of neutrinos in the evolution of primeval adiabatic perturbations, Astrophys. J. 180 (1973) 1.

[51] W. Hu and N. Sugiyama, Small scale cosmological perturbations: An Analytic approach, Astrophys. J. 471 (1996) 542 [astro-ph/9510117] [INSPIRE].

[52] S. Bashinsky and U. Seljak, Neutrino perturbations in CMB anisotropy and matter clustering, Phys. Rev. D 69 (2004) 083002 [astro-ph/0310198] [INSPIRE].

[53] C. Brust, Y. Cui and K. Sigurdson, work in preparation.

[54] C.-P. Ma and E. Bertschinger, Cosmological perturbation theory in the synchronous and conformal Newtonian gauges, Astrophys. J. 455 (1995) 7 [astro-ph/9506072] [INSPIRE].

[55] J. Lesgourgues and S. Pastor, Neutrino cosmology and Planck, New J. Phys. 16 (2014) 065002 [arXiv: 1404.1740] [INSPIRE]. 
[56] Planck collaboration, P.A.R. Ade et al., Planck 2013 results. XVI. Cosmological parameters, Astron. Astrophys. 571 (2014) A16 [arXiv:1303.5076] [INSPIRE].

[57] W. Hu, Lecture Notes on CMB Theory: From Nucleosynthesis to Recombination, arXiv:0802.3688 [INSPIRE].

[58] D.N. Limber, The Analysis of Counts of the Extragalactic Nebulae in Terms of a Fluctuating Density Field. II, Astrophys. J. 119 (1954) 655 [INSPIRE].

[59] Z. Pan, L. Knox and M. White, Dependence of the Cosmic Microwave Background Lensing Power Spectrum on the Matter Density, Mon. Not. Roy. Astron. Soc. 445 (2014) 2941 [arXiv: 1406.5459] [INSPIRE].

[60] L.M. Macri, K.Z. Stanek, D. Bersier, L. Greenhill and M. Reid, A new Cepheid distance to the maser-host galaxy NGC 4258 and its implications for the Hubble Constant, Astrophys. J. 652 (2006) 1133 [astro-ph/0608211] [INSPIRE].

[61] L. Greenhill, E. Humphreys, W. Hu et al., Estimation of the Hubble Constant and Constraint on Descriptions of Dark Energy, arXiv:0902.4255 [INSPIRE].

[62] S. Hannestad, T. Haugboelle and B. Thomsen, Precision measurements of large scale structure with future type-IA supernova surveys, JCAP 02 (2008) 022 [arXiv:0705.0979] [INSPIRE]. 